\title{
Treatment of chronic bronchopleural fistula and recurrent empyema using a latissimus dorsi myocutaneous flap: a case report and literature review
}

\author{
Byungkwon Kang, Yujin Myung \\ Department of Plastic and Reconstructive Surgery, Seoul National University Bundang Hospital, Seoul National University College of \\ Medicine, Seongnam, Korea
}

Bronchopleural fistula is a severe complication with a high mortality rate that occurs after pulmonary resection. Several treatment options have been suggested; however, it is a challenge to treat this condition without recurrence or other complications. In this case report, we describe the successful performance of a pedicled latissimus dorsi myocutaneous flap transfer, with no recurrence or donor site morbidity.

Keywords Bronchial fistula / Pleural diseases / Surgical flaps / Postoperative complications

\author{
Correspondence: Yujin Myung \\ Department of Plastic and \\ Reconstructive Surgery, Seoul \\ National University Bundang Hospital, \\ Seoul National University College of \\ Medicine, 82 Gumi-ro 173beon-gil, \\ Bundang-gu, Seongnam 13620, Korea \\ Tel: +82-31-787-7229 \\ Fax: +82-31-787-4055 \\ E-mail: surgene@snu.ac.kr
}

\section{INTRODUCTION}

Bronchopleural fistula (BPF) is a pathological tract between the airway and the pleural space. Common causes of BPF include pulmonary infection, persistent pneumothorax, chemotherapy, and radiotherapy. BPF is one of the most severe complications that occur after pulmonary resection. Since the reported mortality rate of $\mathrm{BPF}$ is as high as $71 \%$, appropriate management is urgently necessary [1]. In this report, we describe a case of successful closure of BPF using a pedicled latissimus dorsi myocutaneous (LDMC) flap.

\section{CASE}

A 67-year-old male patient was admitted to the department of pulmonology with symptoms of dyspnea, fever, and cough with massive sputum. The patient had a history of non-small cell lung cancer (clinical stage IIIA, T2N2M0) and underwent right upper lobe lobectomy 6 years ago. The patient then received adjuvant chemo-radiotherapy. He had type 2 diabetes mellitus, with a hemoglobin A1c level of 8.8\%, seemed undernourished ( $168 \mathrm{~cm}$ in height, $54 \mathrm{~kg}$ in weight, and a serum albumin level of $2.4 \mathrm{~g} / \mathrm{dL}$ ) and he had smoked for 40 years. After cancer treatment, the patient was repeatedly hospitalized and discharged due to recurrent pneumonia and empyema. Broad-spectrum systemic antibiotic therapy was started from the day of admission. After identification of Mycobacterium abscessus from the sputum, a triple antibiotic regimen (clarithromycin, imipenem/ cilastatin, and amikacin) was started. Under fiberoptic bronchoscopy examination, the patient was found to have a BPF (1.2 $\mathrm{cm}$ in diameter) at the right superior lobar bronchial stump. On computed tomography (CT) imaging, a huge dead space

Copyright $\odot 2021$ The Korean Society of Plastic and Reconstructive Surgeons 
$\left(8.8 \times 7.4 \times 9.1 \mathrm{~cm}^{3}\right)$ was observed in the right upper pleural cavity (Fig. 1). He was then referred to the department of plastic and reconstructive surgery and the department of thoracic sur-
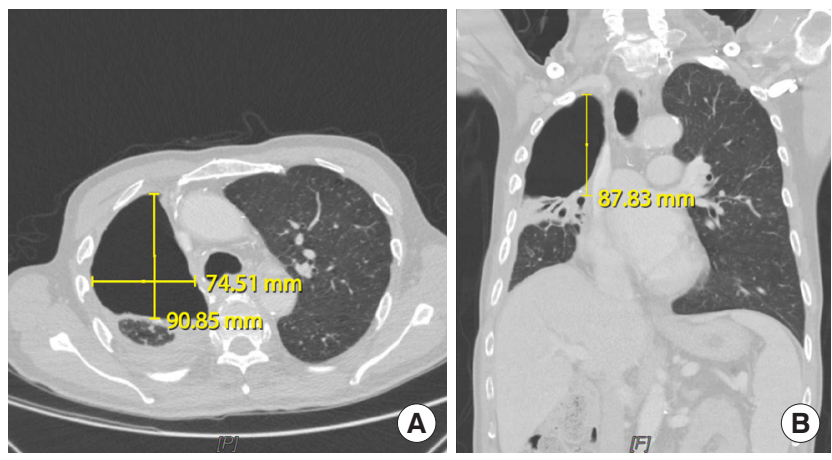

Fig. 1. Preoperative computed tomography image. The dead space in the right upper lobar area and empyema in the right middle lobar area are remarkable. (A) Axial view. (B) Coronal view.
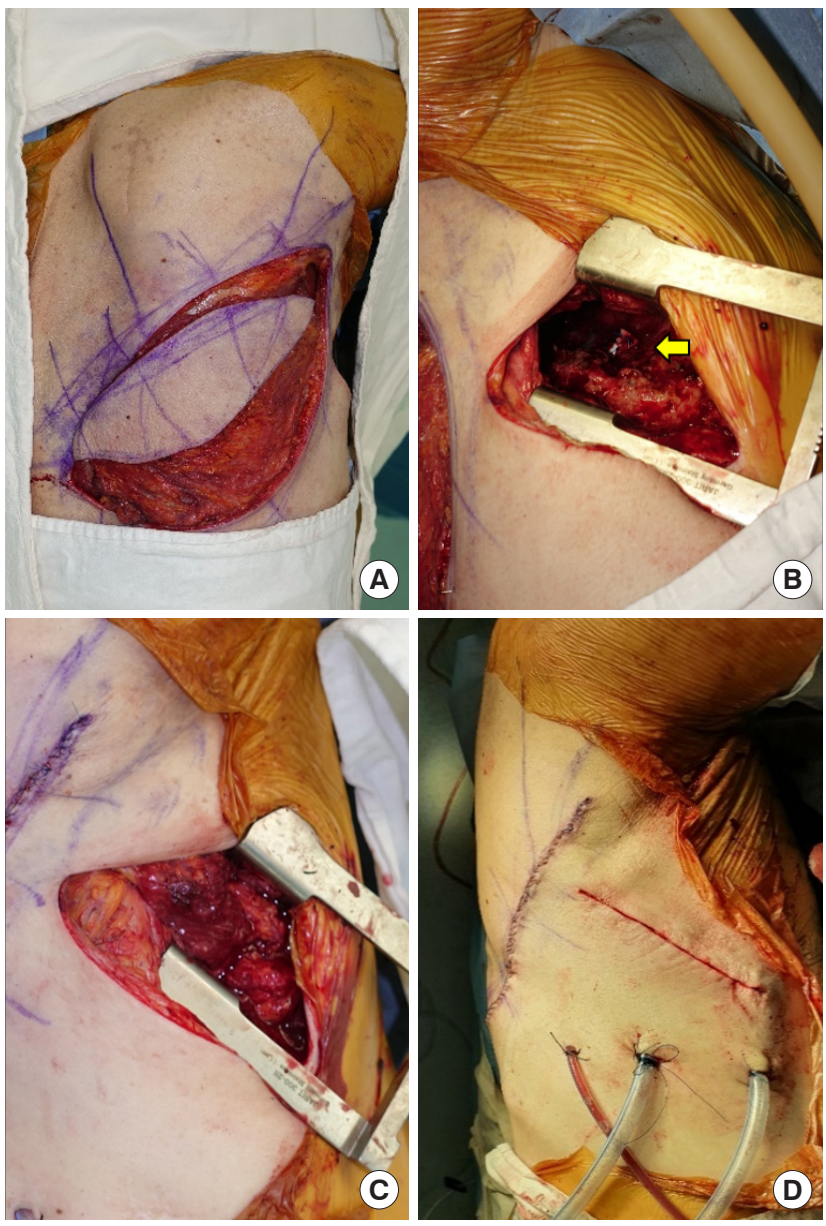

Fig. 2. Intraoperative procedure. (A) A pedicled latissimus dorsi myocutaneous flap was elevated. (B) The pleural cavity and the primarily closed stump of the bronchial fistula (yellow arrow) are observable through the thoracostomy site. (C) The pleural cavity was adequately obliterated by the flap. (D) Postoperative wounds after the insertion of two 28-Fr chest tubes. gery for surgical intervention.

Under general anesthesia, intubated with a double-lumen endotracheal tube, the patient was laid in the left decubitus position. In a collaborative procedure with the thoracic surgery team, thoracostomy was performed through previous thoracostomy scar. Using video-assisted thoracoscopy, the thoracic surgeons identified the BPF site and closed it primarily, followed by decortication of the pleura and debridement of the infected tissues. Next, the plastic surgeons harvested a pedicled LDMC flap with an $8 \times 25 \mathrm{~cm}$ skin paddle. De-epithelization of the skin flap was performed due to its deciduousness. The LDMC flap was transposed into the pleural cavity to obliterate the dead space. Due to the limited view of the pleural space, the fixation of the LDMC flap to the pleural space and BPF stump was performed under a video-assisted thoracoscopic view. Air leakage was excluded by the Valsalva test. The thoracostomy and donor site wounds were closed after inserting 28-Fr chest tubes (Fig. 2 ). The patient was extubated immediately after the operation. On postoperative chest CT, the pleural cavity was completely obliterated (Fig. 3). Postoperative bronchoscopy confirmed a water-tight seal of the fistula. At a 1-month postoperative followup, the patient was in good condition without donor site complications or recurrence of the fistula.

\section{DISCUSSION}

The incidence of BPF after lung surgery has been reported as $1 \%$ to $20 \%$, depending on patients' general condition and surgical techniques [2]. The reported mortality rate ranges from $16 \%$ to $71 \%$ [3]. A small fistula can subside by itself with the insertion of a thoracostomy tube or it can be simply closed by primary sutures [2]. However, as Uramoto and Hanagiri [4] reported, primary closure of a BPF was only successful in $15 \%$ of cases.
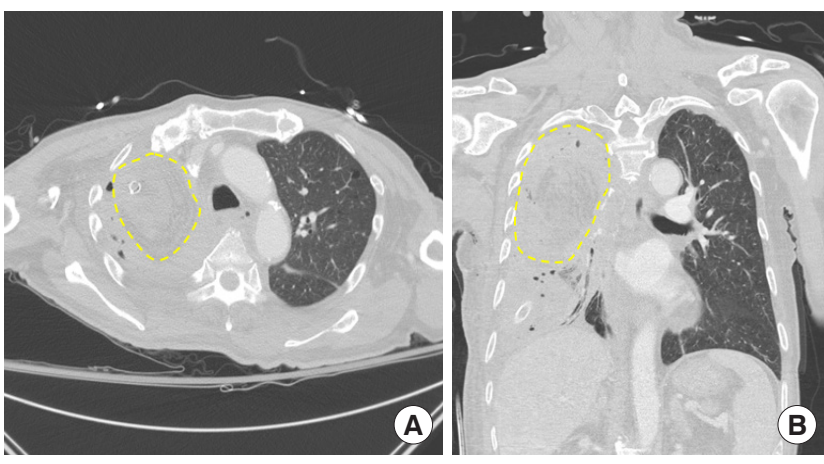

Fig. 3. Postoperative computed tomography image. The pleural dead space was completely obliterated by the latissimus dorsi myocutaneous flap in the right upper lobar area (area covered within the yellow dashed line). (A) Axial view. (B) Coronal view. 
Table 1. Comparisons among flaps used to cover bronchopleural fistula and pleural dead space

\begin{tabular}{lccccc}
\hline Types of flap & Latissimus dorsi & Pectoralis & Serratus anterior & Abdominis & Omentum \\
\hline Coverable lobar defect & Both & Upper & Lower & Lower & Both \\
Volume for covering a large defect & Sufficient & Not sufficient & Not sufficient & Sufficient & Not sufficient \\
Degree of functional/aesthetic morbidity & ++ & +++ & ++ & ++ & + \\
Possible severe complication & Shoulder dysfunction & Aesthetic morbidity & Scapula winging & Hernia & Abdominal complications \\
\hline
\end{tabular}

+ : low, ++: moderate, +++: high.

Therefore, a very large fistula requires a more aggressive intervention.

The severed distal ends of the bronchi after pulmonary resection recovers through a normal wound healing process with fibroblastic proliferation. Avascular necrosis in the peribronchial tissue is one of the main pathogenic mechanisms of BPF after surgical resection [5]. Therefore, a sufficient blood supply from a myocutaneous flap promotes stump healing [6]. Since the first description of muscle transposition for BPF closure by Abrashanoff in 1911, various extra-thoracic flaps using the pectoralis major muscle, serratus anterior muscles, or rectus abdominis muscle have been suggested as surgical treatments of BPF $[7,8]$. However, those muscle flaps have some disadvantages: (1) severe donor site morbidity, including both functional disability (e.g., scapula winging after sacrificing the serratus anterior muscle) and aesthetic deformity (e.g., an asymmetric contour deformity of the chest after sacrificing the pectoralis muscle); (2) insufficient flap volume to fill up the dead space; and (3) and insufficient length of the pedicle to reach the defect. Likewise, a pedicled omental flap has often been described as a favorable option, but it requires additional surgical procedures inside the peritoneal cavity and it also does not provide enough volume. Table 1 presents brief comparisons among the flaps.

Due to its large bulk and relatively long pedicle, the pedicled LDMC flap is a flap of choice $[9,10]$. To obtain maximum volume, we chose to de-epithelize an LDMC flap rather than using a latissimus dorsi muscle flap. Finally, a free flap transfer was not considered since the patient's general condition was not healthy enough to endure a long surgical procedure.

According to Cerfolio [2], the most significant risk factors for BPF after lobectomy were the mode of pulmonary resection, residual carcinoma at the stump or margin, preoperative irradiation, and diabetes mellitus. In this case, the patient underwent lobe resection on the right side, which has the highest prevalence of BPF. The patient was also found to have residual carcinoma tissue on the lobectomy margin. The patient also had a poor general medical condition, with diabetes mellitus, and was malnourished with micro-albuminemia (a serum albumin level of $2.4 \mathrm{~g} / \mathrm{dL}$ ), which is an unfavorable condition for stump healing. Therefore, the patient had almost all the risk factors for BPF.
As Abolhoda et al. [9] emphasized in their article in 2009, prophylactic reinforcement of the bronchial stump using a muscle flap might have been beneficial in this case.

In conclusion, an LDMC flap can be an effective option for the treatment of a very large BPF and recurrent empyema.

\section{NOTES}

\section{Conflict of interest}

No potential conflict of interest relevant to this article was reported.

\section{Ethical approval}

The study was approved by the Institutional Review Board of Seoul National University Bundang Hospital (IRB No. B-2009637-701) and performed in accordance with the principles of the Declaration of Helsinki. The informed consent was exempted by the Institutional Review Board of Seoul National University Hospital because the study does not expose the patient's personal information, so that the publication causes no harm to the patient.

\section{Patient consent}

The patient provided written informed consent for the publication and the use of his images.

\section{Author contribution}

Conceptualization: Y Myung. Data curation: B Kang. Writing original draft: B Kang. Writing - review \& editing: Y Myung.

\section{ORCID}

Byungkwon Kang https://orcid.org/0000-0002-7704-0786

Yujin Myung https://orcid.org/0000-0001-5051-2440

\section{REFERENCES}

1. Smolle-Juttner F, Beuster W, Pinter H, et al. Open-window thoracostomy in pleural empyema. Eur J Cardiothorac Surg 1992;6:635-8.

2. Cerfolio RJ. The incidence, etiology, and prevention of 
postresectional bronchopleural fistula. Semin Thorac Cardiovasc Surg 2001;13:3-7.

3. Asamura H, Naruke T, Tsuchiya R, et al. Bronchopleural fistulas associated with lung cancer operations: univariate and multivariate analysis of risk factors, management, and outcome. J Thorac Cardiovasc Surg 1992;104:1456-64.

4. Uramoto H, Hanagiri T. The development of bronchopleural fistula in lung cancer patients after major surgery: 31 years of experience with 19 cases. Anticancer Res 2011;31: 619-24.

5. Smith DE, Karish AF, Chapman JP, et al. Healing of the bronchial stump after pulmonary resection. J Thorac Cardiovasc Surg 1963;46:548-56.

6. Matsuura M, Fujiwara T, Kataoka K, et al. Two cases of closure of bronchopleural fistula after right pneumonectomy using omental pedicled flap. Jpn J Chest Surg 2009;23:83842.

7. Arnold PG, Pairolero PC. Intrathoracic muscle flaps: an account of their use in the management of 100 consecutive patients. Ann Surg 1990;211:656-60.

8. Kim BJ, Hong IP, Chung CM, et al. Treatment of tuberculous empyema by intrathoracic transposition of a latissimus dorsi muscle flap. Arch Plast Surg 2016;43:117-9.

9. Abolhoda A, Bui TD, Milliken JC, et al. Pedicled latissimus dorsi muscle flap: routine use in high-risk thoracic surgery. Tex Heart Inst J 2009;36:298-302.

10. Heo CY, Min KH, Eun SC, et al. Management of post-lobectomy bronchopleural: cutaneous fistula with a rectus abdominis free flap. J Korean Soc Plast Reconstr Surg 2009;36: 795-8. 